EPJ Web of Conferences 32, 04014 (2012)

DOI: $10.1051 /$ epjconf/20123204014

(C) Owned by the authors, published by EDP Sciences, 2012

\title{
CVD diamond Brewster window: feasibility study by FEM analyses
}

\author{
G. Aiello, G. Grossetti, A. Meier, T. Scherer, S. Schreck, P. Spaeh, D. Strauss and A. Vaccaro
}

Institute for Applied Materials, Karlsruhe Institute of Technology (KIT), P.O. Box 3640, D-76021

Karlsruhe, Germany

\begin{abstract}
Chemical vapor deposition (CVD) diamond windows are a crucial component in heating and current drive (H\&CD) applications. In order to minimize the amount of reflected power from the diamond disc, its thickness must match the desired beam wavelength, thus proper targeting of the plasma requires movable beam reflectors. This is the case, for instance, of the ITER electron cyclotron H\&CD system. However, looking at DEMO, the higher heat loads and neutron fluxes could make the use of movable parts close to the plasma difficult. The issue might be solved by using gyrotrons able to tune the beam frequency to the desired resonance, but this concept requires transmission windows that work in a given frequency range, such as the Brewster window. It consists of a CVD diamond disc brazed to two copper cuffs at the Brewster angle. The brazing process is carried out at about $800^{\circ} \mathrm{C}$ and then the temperature is decreased down to room temperature. Diamond and copper have very different thermal expansion coefficients, therefore high stresses build up during the cool down phase that might lead to failure of the disc. Considering also the complex geometry of the window with the skewed position of the disc, analyses are required in the first place to check its feasibility. The cool down phase was simulated by FEM structural analyses for several geometric and constraint configurations of the window. A study of indirect cooling of the window by water was also performed considering a $\mathrm{HE}_{11}$ mode beam. The results are here reported.
\end{abstract}

\section{Introduction and background}

CVD diamond windows act as confinement barriers in H\&CD applications allowing transmission of high power mm-wave beams at the same time. For example, diamond windows in the ITER electron cyclotron H\&CD system are used to send mm-wave beams between 1 and $2 \mathrm{MW}$ for plasma heating and mode stabilization [1]. To meet several plasma requirements, the beams must be deployed at different positions inside the plasma. This can be achieved by sending a fixed frequency beam via moveable mirrors or a beam at different frequencies. In the first case, a conventional single disc window is used and thus the disc thickness must be in resonance condition in order to minimize the power reflection. This means that the thickness of the disc must be an integer multiple of half the wavelength of the beam inside the material. Moveable mirrors are so used to deploy the beam at different locations, as in the ITER electron cyclotron H\&CD system [2].

In the second case, using gyrotrons able to tune the beam frequency to the desired resonance in a given range (e.g. 105-140 GHz) [3], the beam is directly sent to the correspondent resonant magnetic 
flux surface. This method would be much faster than the first one to fulfil several plasma requirements because no need for mechanical movement of parts, and furthermore it also would avoid using moveable components close to the plasma. On the other hand, this method requires broadband window solutions such as the double disc window [4] or the Brewster window. The Brewster window consists of a CVD diamond disc brazed to two oxygen-free high conductivity (OFHC) copper cuffs at the Brewster angle. In theory, this window does not have power reflection from the disc varying the frequency of the beam. The choice of the disc thickness would be thus affected by the stress profiles arising from window construction and normal operation only. In reality, varying the frequency, the window has a little percentage of power reflection due to some factors and so the choice of the disc thickness depends also on the most important desired frequencies of the beam $[5,6]$.

The geometry of the Brewster window is quite complex because of the skewed position of the disc and, furthermore, it involves brazing of diamond and copper which have very different thermal expansion coefficients. The brazing process is carried out at $800^{\circ} \mathrm{C}$ and thus the cool down phase to room temperature results in high stresses that might lead to failure of the disc. This paper presents a feasibility study of the Brewster window by FEM structural analyses considering several geometric and constraint configurations. A study of an indirectly water-cooled configuration is also presented, considering the power absorption in the diamond disc due to a $\mathrm{HE}_{11}$ mode beam.

\section{Methods}

\subsection{Reference configuration}

\subsubsection{Geometry}

A FEM model of the Brewster window was set up as reference case and it is shown in Figure 1a. The CVD diamond disc has an elliptical shape with major axis of $160 \mathrm{~mm}$, minor axis of $80 \mathrm{~mm}$ and thickness of $1.9 \mathrm{~mm}$. The OFHC copper cuffs are cylindrical with inner diameter of $50 \mathrm{~mm}$, thickness of $1 \mathrm{~mm}$ and total length of $200 \mathrm{~mm}$. Symmetry was assumed along the major axis of the disc allowing the analysis of half structure. The disc is brazed to the cuffs at the Brewster angle given by $\theta_{B}=\arctan \left(\sqrt{\varepsilon_{r}}\right)$, where $\varepsilon_{r}$ is the dielectric constant of diamond. Its value is 5.67 in the range of the mm-waves and so it turns out that $\theta_{B}=67.22^{\circ}$. The coordinate system has the origin in the center of mass of the disc with $z$-axis oriented along the axis of the cuffs and $x$-axis along the minor axis of the disc. The Brewster angle is formed between the normal to the disc in the origin and the axis of the cuffs.

\subsubsection{Material properties}

No brazing material was considered in this study. The properties for CVD diamond and OFHC copper were taken respectively from [7, 8] and [9]. Poisson's ratio for both materials and density, Young's modulus for diamond were assumed as constant properties. Secant coefficient of thermal expansion, thermal conductivity, heat capacity for both materials and density, Young's modulus for copper were instead assumed as temperature dependent properties between $20^{\circ} \mathrm{C}$ and $800^{\circ} \mathrm{C}$. Non linear properties were also used for copper to take account of the plastic behaviour of the cuffs. The multilinear isotropic hardening model in ANSYS was used to simulate the copper plasticity and thus plastic strain versus stress curves were defined at different temperatures in the range of interest.

\subsubsection{Boundary condition and load}

The cool down phase of the brazing process was simulated by static structural analysis in ANSYS Workbench V13. A fixed support was applied to the two ends of the copper cuffs as boundary condition. The equilibrium temperature was $800^{\circ} \mathrm{C}$ and a $20^{\circ} \mathrm{C}$ thermal condition was applied to the 
structure. The ramped load was specified in a single load step and, since the analysis is non linear, substeps were used to apply gradually the load in order to obtain an accurate solution.

a)

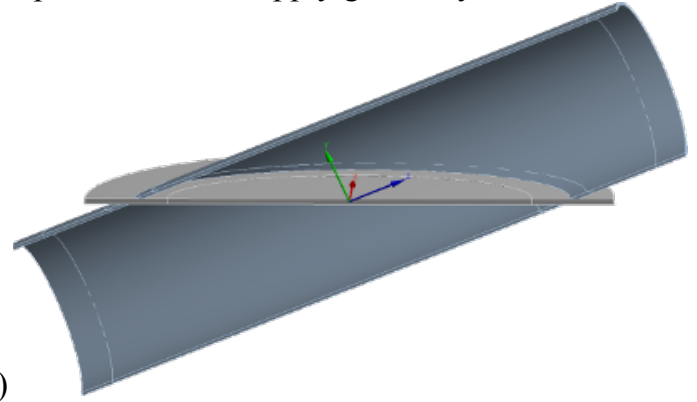

b)

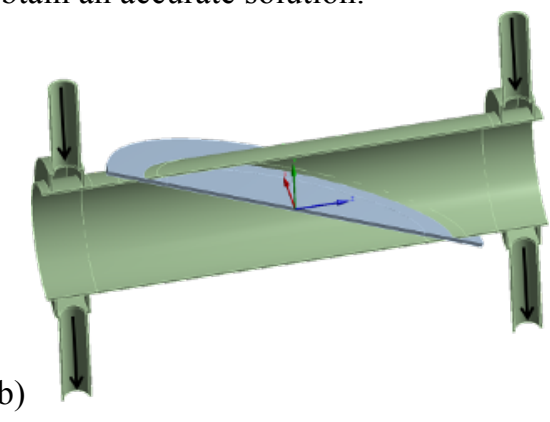

Fig. 1. Reference configuration (a) and indirectly water-cooled configuration (b) of the Brewster window. The arrows in (b) show the inlet and outlet channels.

\subsection{Geometric and constraint configurations}

With respect to the reference case, a parameters impact analysis was then carried out by varying the thickness of the cuffs and the disc and the aspect ratio of the disc. The thickness varies from 0.8 to 2 $\mathrm{mm}$ for the cuffs and from 1 to $2.5 \mathrm{~mm}$ for the disc. The range for the disc includes optimum thicknesses for beam frequencies of interest such as $1.6 \mathrm{~mm}$ for $120-130 \mathrm{GHz}$ and $1.9 \mathrm{~mm}$ for operation near $105 \mathrm{GHz}$ and $140 \mathrm{GHz}$ [6]. In the reference case, the disc has an aspect ratio of 2 . The major axis of $160 \mathrm{~mm}$ is maybe too big for a real manufacturing of the diamond disc, so other two aspect ratios, 1.4 and 2.33, were considered with major axis of $140 \mathrm{~mm}$ (the possible minimum value, being $50 \mathrm{~mm}$ the diameter of the cuffs) and minor axis respectively of $100 \mathrm{~mm}$ and $60 \mathrm{~mm}$.

Up to now, both the ends of the cuffs were fixed as boundary condition. Other two constraint configurations were also investigated with respect to the reference one in order to compare different ways of carrying out the brazing process. Looking at the coordinate system in Figure 1a, one configuration (named configuration 1) has the lower end of the cuffs fixed and the upper one free to move along the axis of the cuffs only, while the other configuration (named configuration 2) has always the lower end fixed and the upper one without constraints.

\subsection{Indirectly water-cooled configuration}

Subsequently, an indirect cooling circuit of OFHC copper was added to the reference geometric model to evaluate maximum temperatures and thermal stresses in the window due to the power absorption in the disc, considering a $\mathrm{HE}_{11}$ mode beam. Figure $1 \mathrm{~b}$ reports the reference model with the cooling circuit of $12 \times 8 \mathrm{~mm}$ rectangular cross section and inlet and outlet channels of $10 \mathrm{~mm}$ diameter. First, a CFD analysis was performed in ANSYS CFX V13 to calculate the heat exchange coefficient along the cooling path of the structure. The CFD model consists of the fluid domain only and water properties defined in the CFX's library were used. A mass flow rate of $10 \mathrm{~kg}$ per minute was considered at each inlet, thus a rate of $0.0833 \mathrm{~kg} \mathrm{~s}^{-1}$ was applied since the model is symmetric. A reference pressure of $0 \mathrm{~Pa}$ was instead applied to the outlets. The inlet water has a reference temperature of $20^{\circ} \mathrm{C}$, which can be considered constant along the cooling path.

A thermal analysis was then carried out by interpolating the heat exchange coefficient at the cooling interface and applying a heat generation load to the disc according to the $\mathrm{HE}_{11}$ pattern. The applied volumetric power density distribution $q^{\prime \prime \prime}\left[\mathrm{W} \mathrm{m} \mathrm{m}^{-3}\right]$ was given by the law $q^{\prime \prime \prime}(r)=A \cdot\left[J_{0}(x)\right]^{2}$ being $x=2.405 \cdot(r / a)$, where $A$ is the normalizing constant, $r$ is the radial coordinate and $a$ is the radius of the cuffs. The Bessel function $J_{0}(x)$ was expressed by its Taylor series expansion around $x$ $=0$ and the law was normalized for several values of absorbed power in the Brewster disc. Temperature distributions of the window were calculated by running thermal analyses with absorbed 
power of $0.2,0.4,0.6,0.8$ and $1 \mathrm{~kW}$. Considering a beam power of $1 \mathrm{MW}$, the previous values correspond respectively to fractions of $0.02,0.04,0.06,0.08$ and $0.1 \%$ of the transmitted power.

Finally, the temperature gradient corresponding to the absorbed power of $0.4 \mathrm{~kW}$ was applied to the window in a second load step of the structural analysis of the reference configuration. The purpose was to investigate how the temperature gradient, due to the power absorption in the disc, affects the stress field generated in the disc during the cool down phase of the brazing process.

\section{Results and discussion}

\subsection{Reference configuration}

In the reference case, both the ends of the cuffs are fixed and therefore the distribution of the stresses in the Brewster window, shown in Figure 2, is antisymmetric with respect to the center of mass of the disc. The first principal stress is generally in the range $30-75 \mathrm{MPa}$ along the contact region between the disc and the cuffs and has a maximum of $137.4 \mathrm{MPa}$, located at the tip of the disc in the contact region with the longer side of the cuffs. The cuffs experience plastic behaviour, in fact the equivalent stress varies between 50 and $75 \mathrm{MPa}$ (yield stress of OFHC copper is $45 \mathrm{MPa}$ at $20^{\circ} \mathrm{C}$ [9]) in the most part of the cuffs and has maxima values in the range 103.1-110.6 MPa, located close to the area of maximum principal stress and to the support.

The very different thermal expansion coefficient between copper and diamond (ratio of 16 to 1 at $20^{\circ} \mathrm{C}$, respectively) and the fixed support cause these stress profiles. The longer side of the cuffs is the part where the maximum deformation (shown in \$3.2) occurs and thus the higher stresses in the disc and the cuffs are located in correspondence of it. However, the maximum principal stress is below the permissible stress of the diamond which is $150 \mathrm{MPa}$ (ultimate stress is $450-500 \mathrm{MPa}$ [10]) and the maximum equivalent stress is below the ultimate stress of $\mathrm{OFHC}$ copper $\left(250 \mathrm{MPa}\right.$ at $20^{\circ} \mathrm{C}$ [9]).
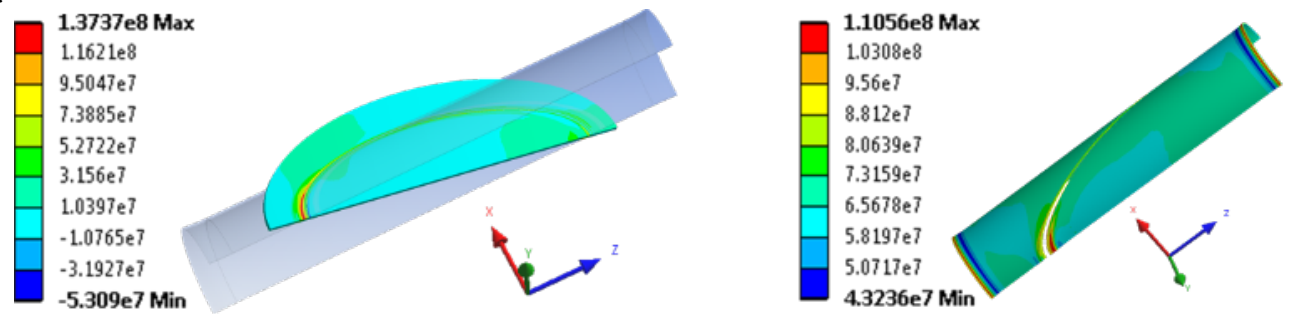

Fig. 2. First principal stress distribution on the top side of the diamond disc (left) and von-Mises stress distribution on the copper cuffs (right) in the reference case. Values are in $\mathrm{Pa}$.

\subsection{Geometric and constraint configurations}

Figure 3 shows the maxima stresses as functions of the thickness of the cuffs and the disc. The first principal stress grows significantly as the cuff thickness increases or the disc thickness decreases, reaching values well above the permissible stress. In the first case, the cuffs become stiffer and the contact region larger, thus more severe stress profiles are generated in the disc while in the second case, the reason is that the disc becomes less stiff. Values of cuff thickness above $1.2 \mathrm{~mm}$ and of disc thickness below $1.7 \mathrm{~mm}$ are not recommended. On the contrary, the equivalent stress is not affected by the variation of the thickness since the cuffs are in plastic field. Then, the aspect ratio of the disc affects the first principal stress profile by less than $3 \%$.

Finally, the other two constraint configurations (configurations 1 and 2) lead to a maximum first principal stress in the disc of $181 \mathrm{MPa}$, greater than that obtained in the reference case and also above the permissible stress. In addition, the disc has wide areas with stress values in the range 70$115 \mathrm{MPa}$. This difference might be explained looking at the Figure 4 where the reference configuration is compared to the configuration 1. In configuration 1 , because of the boundary 
condition, the plastic deformation occurs only in the part of the cuffs near the disc but the deformation is big and the deformation mechanism would lead to severe stresses in the disc. In contrast, in the reference case due to the fixed support, the deformation is more limited, occurring only in the plane perpendicular to the $z$-axis, but the cuffs are wholly plastically deformed. This would result in a more relaxed stress profile in the diamond disc of the reference case. The deformation in the reference configuration is antisymmetric with a maximum of $1.23 \mathrm{~mm}$ and the disc rotates of about $-0.35^{\circ}$ with respect to $x$-axis. In configuration 1 , the window shifts to the right side with maximum deformation of $2.83 \mathrm{~mm}$ and the disc moves of about $2 \mathrm{~mm}$. The considerations done for the configuration 1 are also valid for the configuration 2, but here the maximum deformation amounts to $3.32 \mathrm{~mm}$ and the disc moves of about $3.2 \mathrm{~mm}$.
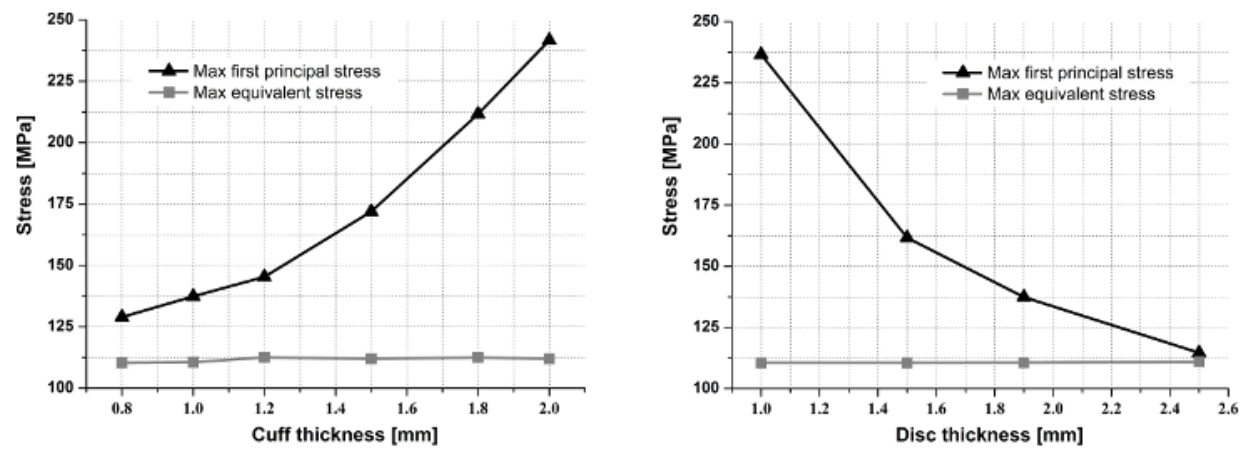

Fig. 3. Maxima first principal stress and von-Mises stress as functions of the cuff thickness (left) and disc thickness (right).

a)

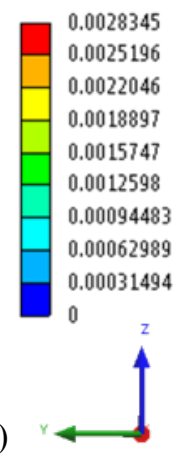

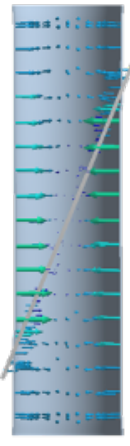

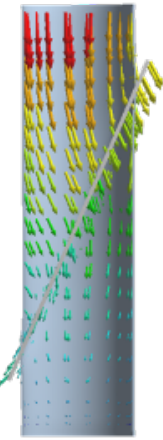

b)

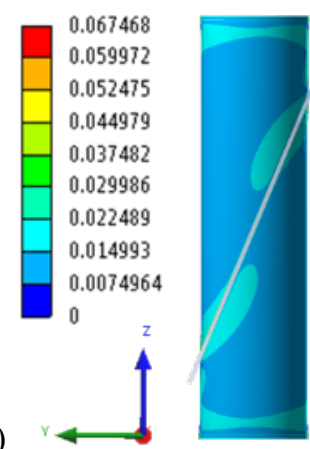

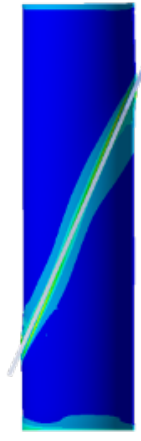

Fig. 4. Comparison of the deformation mechanism (a) and equivalent plastic strain distribution (b) between the reference configuration on the left and the configuration 1 (upper cuff end free to move in the $z$-direction only) on the right. Values are in $\mathrm{m}$ (a) and dimensionless (b).

\subsection{Indirectly water-cooled configuration}

The heat exchange coefficient calculated by CFD analysis and interpolated at the cooling interface of the configuration shown in Figure $1 \mathrm{~b}$ varies mainly from 60000 to $80000 \mathrm{~W} \mathrm{~m}^{-2} \mathrm{~K}^{-1}$. The maximum temperature is reached in the center of the disc and it is shown as a function of the absorbed power in the disc in Figure 5a. It can be noted that going over the power value of $0.4 \mathrm{~kW}(0.04 \%$ of a $1 \mathrm{MW}$ power beam), the maximum temperature reaches values well above $200^{\circ} \mathrm{C}$. The cooling circuit is cylindrical and it is very far from the heat source because of the skewed position of the disc, so the heat is transmitted by conduction for a long path in the copper cuffs giving rise to high temperatures. A more effective cooling circuit would be a circuit of elliptical shape which follows the Brewster disc, but maybe this option would give some manufacturing issues. 
When the temperature gradient, corresponding here to the absorbed power of $0.4 \mathrm{~kW}$, is applied to the window in a second load step of the structural analysis of the reference configuration, the stress profile shown in Figure $5 \mathrm{~b}$ is obtained for the disc. The maximum first principal stress decreases from 137.4 to $98.7 \mathrm{MPa}$, located now at the tip of the disc in the contact region with the shorter side of the cuffs. However, the distribution becomes slightly more severe since now the stress along the contact region between the disc and the cuffs is in the range 60-75 MPa for the most part and an area with stresses in the range 30-50 MPa appears in the outer side of the disc. In conclusion, the stress field generated in the disc by the cool down phase of the brazing process and the applied temperature gradient is well below the permissible stress of the diamond.

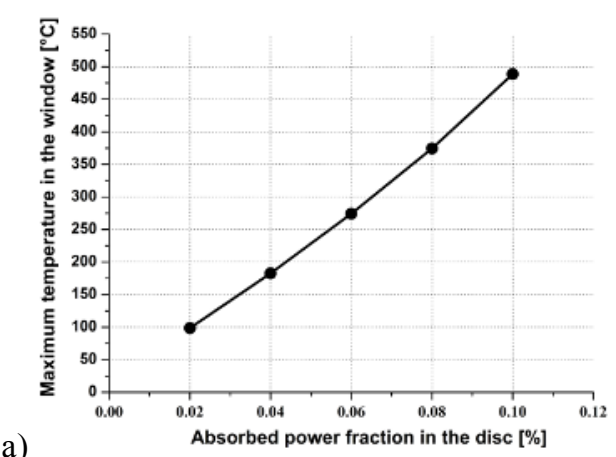

a)

Fig. 5. Maximum temperature (a) in the indirectly water-cooled configuration as a function of the absorbed power fraction in the diamond disc with respect to a $1 \mathrm{MW}$ power beam. Comparison (b) of the first principal stress distribution on the top side of the disc (antisymmetric distribution) between the reference case on the left and the reference case with the applied second load step on the right. Values are in Pa.

\section{Conclusions}

FEM structural analyses showed that the CVD diamond Brewster window is a feasible broadband window solution for H\&CD applications. Values of the cuff thickness greater than $1.2 \mathrm{~mm}$ and of the disc thickness less than $1.7 \mathrm{~mm}$ are not recommended.

\section{References}

1. T. Scherer et al., Experimental analysis of the inserted waveguide CVD diamond window prototype for the ITER ECRH upper launcher, conf. proc. 36th IRMMW-THz, Houston, 2011.

2. D. Strauss et al., Preliminary Design of the ITER ECH Upper Launcher, conf. proc. 38th ICOPS, Chicago, 2011.

3. D. Wagner et al., J. Infrared Milli Terahz Waves 32, 274-282 (2011).

4. R. Heidinger et al., Low power mm-wave transmission characteristics of a frequency tuneable double disk CVD-diamond window, conf. proc. 32nd IRMMW-THz, Cardiff, UK, 2007.

5. J. Flamm et al., Measurements on a Brewster Window for a High Power Gyrotron, conf. proc. 31st IRMMW-THz, Shanghai, China, 2006.

6. X. Yang et al., Int. J. Infrared Milli Waves 24, 5 (2003).

7. D.C. Harris, Infrared Window and Dome Materials (SPIE, Washington, 1992).

8. Y.S. Touloukian et al., Thermophysical Properties of Matter - Vol. 5.

9. M. Merola et al., ITER plasma facing component materials database in ANSYS format, ITER Doc. G17 MD 71 96-11-19 W 0.1, 1997.

10. M. Thumm, State of the Art of High Power Gyro-Devices and Free Electron Masers, KIT Scientific Reports 7575, Update 2010. 